https://helda.helsinki.fi

\title{
A former career as a male elite athlete-does it protect against type 2 diabetes in later life?
}

\section{Laine, Merja K.}

2014-02

Laine , M K, Eriksson , J G , Kujala , U M , Wasenius , N S , Kaprio , J , Backmand , H M , Peltonen , M , Mertsalmi , T H \& Sarna , S 2014 , ' A former career as a male elite athlete-does it protect against type 2 diabetes in later life? ' , Diabetologia , vol. 57 , no. 2 , pp. 270-274 . https://doi.org/10.1007/s00125-013-3105-8

http://hdl.handle.net/10138/298426

https://doi.org/10.1007/s00125-013-3105-8

publishedVersion

Downloaded from Helda, University of Helsinki institutional repository.

This is an electronic reprint of the original article.

This reprint may differ from the original in pagination and typographic detail.

Please cite the original version. 


\title{
A former career as a male elite athlete-does it protect against type 2 diabetes in later life?
}

\author{
Merja K. Laine • Johan G. Eriksson • Urho M. Kujala • \\ Niko S. Wasenius • Jaakko Kaprio • Heli M. Bäckmand • \\ Markku Peltonen • Tuomas H. Mertsalmi • Seppo Sarna
}

Received: 30 July 2013 / Accepted: 15 October 2013 /Published online: 21 November 2013

(C) Springer-Verlag Berlin Heidelberg 2013

\begin{abstract}
Aims/hypothesis The aim of this study was to determine the prevalence of impaired glucose regulation in male Finnish former elite athletes and age- and area-matched controls. We hypothesised that vigorous physical activity during young adulthood protects from disturbances in glucose regulation in later life.

Methods In 2008, 392 former male elite athletes (mean age $72.7 \pm 6.1$ years) and 207 controls (mean age $71.6 \pm 5.6$ years) participated in a clinical study (participation rate: 50.6\%). The former athletes were divided into three groups based on their active career sport: endurance, mixed and power sports.
\end{abstract}

Electronic supplementary material The online version of this article (doi:10.1007/s00125-013-3105-8) contains peer-reviewed but unedited supplementary material, which is available to authorised users.

M. K. Laine $(\bowtie) \cdot$ J. G. Eriksson $\cdot$ N. S. Wasenius

Department of General Practice and Primary Health Care, University of Helsinki, Tukholmankatu 8 B, PL 20, 00014 Helsinki, Finland

e-mail: merja.k.laine@helsinki.fi

\section{K. Laine}

Vantaa Health Center/Network of Academic Health Centers,

University of Helsinki, Helsinki, Finland

\section{J. G. Eriksson • M. Peltonen}

Division of Welfare and Health Promotion, Department of Chronic

Disease Prevention, Diabetes Prevention Unit, National Institute for

Health and Welfare, Helsinki, Finland

\section{J. G. Eriksson}

Folkhälsan Research Centre, Helsinki, Finland

\section{J. G. Eriksson}

Unit of General Practice, Helsinki University Central Hospital,

Helsinki, Finland

J. G. Eriksson

Vasa Central Hospital, Vasa, Finland
Participants without a history of diabetes $(n=537)$ underwent a 2 h 75 g OGTT. Current volume of leisure-time physical activity (LTPA) was determined by self-reported questionnaires and expressed in metabolic equivalent hours (MET-h). Data on reimbursable diabetes medication from participants and non-participants was obtained from the register of the Finnish Social Insurance Institution.

Results Compared with the controls, the former elite athletes had a significantly lower risk of type 2 diabetes (OR 0.72 , $95 \%$ CI $0.53,0.98)$. The risk of type 2 diabetes decreased with increased LTPA volume (OR 0.98, 95\% CI 0.97, 0.99 per 1 MET-h/week). The former elite athletes also had a

\author{
U. M. Kujala \\ Department of Health Sciences, University of Jyväskylä, \\ Jyväskylä, Finland \\ J. Kaprio $\cdot$ H. M. Bäckmand $\cdot$ S. Sarna \\ Department of Public Health, University of Helsinki, \\ Helsinki, Finland \\ J. Kaprio \\ Department of Mental Health and Substance Abuse Services, \\ National Institute for Health and Welfare, \\ Helsinki, Finland \\ J. Kaprio \\ Institute for Molecular Medicine, University of Helsinki, \\ Helsinki, Finland \\ H. M. Bäckmand \\ Health and Social Welfare Department, City of Vantaa, \\ Vantaa, Finland \\ T. H. Mertsalmi \\ Faculty of Medicine, University of Helsinki, Helsinki, Finland
}


significantly lower risk of impaired glucose tolerance (IGT) than the controls (OR $0.58,95 \%$ CI $0.38,0.87$ ).

Conclusions/interpretation A former career as an elite athlete protected from both type 2 diabetes and IGT in later life. In addition, the volume of current LTPA was inversely associated with the prevalence of type 2 diabetes.

Keywords Athlete $\cdot$ Impaired fasting glucose $\cdot$ Impaired glucose tolerance $\cdot$ Physical activity $\cdot$ Type 2 diabetes

\section{Abbreviations \\ IFG Impaired fasting glucose \\ IGT Impaired glucose tolerance \\ LTPA Leisure-time physical activity \\ MET-h Metabolic equivalent hours}

\section{Introduction}

The burden of type 2 diabetes is increasing worldwide. The underlying causes are multifactorial, but overweight, physical inactivity and genetic predisposition are believed to be major underlying factors [1]. Regular physical activity is known to reduce the risk of a number of health outcomes, including type 2 diabetes [2,3]. However, less is known about the effect of vigorous physical activity during young adulthood on later diabetes risk.

In 1985, a questionnaire-based study was initiated to study Finnish former male elite athletes and age- and area-matched controls. The questionnaires were repeated in the years 1995 , 2001 and 2008. In addition, a clinical study was conducted in 2008.

Here we present data from the clinical study in relation to type 2 diabetes and other disturbances in glucose regulation.

\section{Methods}

Participants The study population consists of male former elite athletes who represented Finland at least once in a international competition (inter-country competition or higher) between the years 1920 and 1965 and their age- and areamatched controls. The controls were classified as healthy at the medical examination, which all Finnish men undergo at about 20 years of age as part of the national military service. Former athletes were divided into three groups: endurance sports (long- and middle-distance running, cross-country skiing), mixed sports (football; ice hockey; basketball; track and field: jumping, sprinting and hurdling events, decathlon) and power sports (boxing, wrestling, weight-lifting, track and field throwing events). The division was made according to the type of training needed to achieve optimal results.
In 1985, a questionnaire was sent to 1,518 former athletes and 1,010 controls, and further questionnaires were sent out in 1995 and 2001. In 2008, an invitation to participate in a clinical study was sent to all former athletes $(n=747$, of whom 392 participated) and controls $(n=436$, of whom 207 participated) who were still alive and who had answered at least one of the questionnaires sent in 1985, 1995 and 2001. The clinical study included a physical examination, laboratory tests and questionnaires.

Data on reimbursable diabetes medication was obtained from the register of the national Finnish Social Insurance Institution [4].

The Ethics Committee of the Hospital District of Helsinki and Uusimaa approved the study, and all participants provided written informed consent.

Laboratory analyses Glucose was measured according to the WHO 1999 guidelines [5] after participants had fasted for $12 \mathrm{~h}$. Participants without a history of diabetes underwent a standard $2 \mathrm{~h} 75 \mathrm{~g}$ OGTT. A blood sample was drawn $2 \mathrm{~h}$ after the ingestion of the $300 \mathrm{ml}$ solution, containing $75 \mathrm{~g}$ anhydrous glucose and $1.6 \mathrm{~g}$ citric acid. Plasma glucose was analysed using a dehydrogenase method (ABX Diagnostics, Montpellier, France). Type 2 diabetes, impaired fasting glucose (IFG) and impaired glucose tolerance (IGT) were diagnosed according to WHO criteria from year 1999 [5]. Any participant with both IFG and IGT was classified as having IGT. Three participants had missing OGTT data.

Anthropometric data and assessment of smoking BMI was calculated as measured weight $(\mathrm{kg})$ divided by height squared $\left(\mathrm{m}^{2}\right)$. The participants' smoking habits were assessed by a self-administered questionnaire. Participants were considered smokers if they had smoked over 100 cigarettes in their lifetime and still smoked at least one cigarette daily or almost daily or had quit smoking less than 6 months ago. Data on smoking habits were missing for nine participants.

Assessment of leisure-time physical activity The leisure-time physical activity (LTPA) questionnaire asked questions on the average intensity, duration and frequency of activity during the previous 3 months (see Electronic supplementary material [ESM] Methods). The volume of LTPA was calculated by multiplying the intensity (in metabolic equivalents), duration and frequency of LTPA and is expressed in metabolic equivalent hours (MET-h). This method has been validated against detailed physical activity interview. LTPA data were missing for 15 participants.

Family history of type 2 diabetes Data on family history of type 2 diabetes were missing for 761 participants $(64.3 \%$ of the original study cohort) and so the effect of family history could not be investigated in this study. 
Statistical analyses Data are reported as means \pm SD. Percentage differences were tested using cross-tabulation and the $\chi^{2}$ test. Logistic regression was used to estimate the OR and 95\% CI. Means were compared by one-way ANOVA, and post hoc tests by Bonferroni correction. The general linear model (GLM) was used to adjust for other variables. Statistical analyses were carried out using IBM SPSS, version 21.0 (IBM, Armonk, NY, USA). A $p$ value of less than 0.05 was considered statistically significant.

\section{Results}

Of the original study cohort $(n=1,183), 50.6 \%(n=599)$ participated and $49.4 \%(n=584)$ did not. The non-participants were older (mean age 74.9 [SD 7.3]years) than the participants (mean age 72.3 [SD 5.9]years). More non-participants $(12.8 \%)$ than participants $(8.3 \%)$ had reimbursable diabetes medication $(p=0.012)$. Patient characteristics are shown in Table 1. Current LTPA was $53.2 \%$ higher for the former athletes than for the controls (31.4 vs 20.5 MET-h/week, $p<$ $0.001)$. Fewer former athletes than controls smoked $(5.1 \%$ vs $10.4 \%, p=0.016$ ).

The age-adjusted prevalence of type 2 diabetes (self-reported and screen-detected combined) tended to be lower among the former athletes than among the controls (OR $0.68,95 \% \mathrm{CI}$ $0.45,1.01)$. The age-adjusted prevalence of IGT was significantly lower among the former athletes than among the controls (OR $0.58,95 \%$ CI $0.38,0.87$ ). Adjusting for smoking did not influence the results.

Based on data on both self-reported and screen-detected type 2 diabetes as well as reimbursable diabetes medication in the whole study cohort, the prevalence of type 2 diabetes among the former athletes was significantly lower than among the controls (age-adjusted OR $0.72,95 \%$ CI $0.53,0.98$ ). The former athletes in the endurance group had the lowest prevalence of type 2 diabetes (compared with the controls OR 0.39 ,

Table 1 Distribution of age, BMI, leisure-time physical activity and abnormalities in glucose regulation among the participants in the clinical study in the year 2008

\begin{tabular}{|c|c|c|c|c|c|}
\hline & Endurance $(n=64)$ & Mixed $(n=221)$ & Power $(n=107)$ & All athletes $(n=392)$ & Controls $(n=207)$ \\
\hline Age (years) & $75.3 \pm 5.5^{* * *}$ & $71.9 \pm 6$ & $72.8 \pm 6.2$ & $72.7 \pm 6.1 *$ & $71.6 \pm 5.6$ \\
\hline BMI $\left(\mathrm{kg} / \mathrm{m}^{2}\right)$ & $25.0 \pm 3.3 * *$ & $26.1 \pm 3.3$ & $28.2 \pm 4.7 * *$ & $26.5 \pm 3.9$ & $26.8 \pm 3.4$ \\
\hline LTPA (MET-h/week) ${ }^{\mathrm{a}}$ & $42.8 \pm 36.6^{* * *}$ & $31 \pm 27.3^{* * *}$ & $25.2 \pm 23.2$ & $31.4 \pm 28.54^{* * *}$ & $20.5 \pm 21.7$ \\
\hline SRT2D & $4.7(3)$ & $7.7(17)$ & $9.3(10)$ & $7.7(30)$ & $15.5(32)$ \\
\hline No adjusting & $0.27(0.08,0.91)$ & $0.46(0.24,0.85)$ & $0.56(0.27,1.20)$ & $0.45(0.27,0.77)$ & \\
\hline $\mathrm{ST} 2 \mathrm{D}^{\mathrm{b}}$ & $9.4(6)$ & $11.4(25)$ & $13.3(14)$ & $11.6(45)$ & $10.1(21)$ \\
\hline No adjusting & $0.91(0.35,2.35)$ & $1.12(0.61,2.08)$ & $1.35(0.66,2.77)$ & $1.15(0.66,1.98)$ & \\
\hline $\mathrm{T} 2 \mathrm{D}^{\mathrm{b}}$ & $14.1(9)$ & $19.1(42)$ & $22.9(24)$ & $19.3(75)$ & $25.6(53)$ \\
\hline No adjusting & $0.48(0.22,1.03)$ & $0.69(0.43,1.08)$ & $0.86(0.50,1.50)$ & $0.69(0.46,1.04)$ & \\
\hline Adjusting for age & $0.43(0.20,0.94)$ & $0.68(0.43,1.08)$ & $0.84(0.48,1.46)$ & $0.68(0.45,1.01)$ & \\
\hline Adjusting for BMI & $0.60(0.27,1.31)$ & $0.76(0.47,1.21)$ & $0.70(0.39,1.24)$ & $0.72(0.47,1.08)$ & \\
\hline Adjusting for age and BMI & $0.53(0.28,1.17)$ & $0.75(0.47,1.20)$ & $0.66(0.36,1.18)$ & $0.69(0.45,1.04)$ & \\
\hline $\mathrm{IFG}^{\mathrm{b}}$ & $25.0(16)$ & $22.3(49)$ & $22.9(24)$ & $22.9(89)$ & $17.4(36)$ \\
\hline No adjusting & $1.58(0.81,3.10)$ & $1.36(0.81,2.20)$ & $1.41(0.79,2.51)$ & $1.41(0.92,2.17)$ & \\
\hline Adjusting for age & $1.91(0.96,3.80)$ & $1.39(0.86,2.25)$ & $1.48(0.83,2.66)$ & $1.48(0.96,2.29)$ & \\
\hline Adjusting for BMI & $1.54(0.78,3.04)$ & $1.35(0.83,2.18)$ & $1.43(0.80,2.57)$ & $1.40(0.91,2.16)$ & \\
\hline Adjusting for age and BMI & $1.85(0.92,3.71)$ & $1.37(0.84,2.22)$ & $1.52(0.84,2.74)$ & $1.47(0.95,2.27)$ & \\
\hline $\mathrm{IGT}^{\mathrm{b}}$ & $21.9(14)$ & $17.3(38)$ & $20.0(21)$ & $18.8(73)$ & $26.6(55)$ \\
\hline No adjusting & $0.77(0.40,1.51)$ & $0.58(0.36,0.92)$ & $0.69(0.39,1.22)$ & $0.64(0.43,0.95)$ & \\
\hline Adjusting for age & $0.60(0.30,1.19)$ & $0.55(0.34,0.88)$ & $0.63(0.35,1.12)$ & $0.58(0.38,0.87)$ & \\
\hline Adjusting for BMI & $0.80(0.41,1.58)$ & $0.59(0.37,0.94)$ & $0.67(0.38,1.19)$ & $0.64(0.43,0.96)$ & \\
\hline Adjusting for age and BMI & $0.62(0.31,1.25)$ & $0.56(0.35,0.90)$ & $0.60(0.33,1.08)$ & $0.58(0.38,0.88)$ & \\
\hline
\end{tabular}

Results are presented as means $\pm \mathrm{SD}, \mathrm{OR}(95 \% \mathrm{CI})$ or $\%(n)$

${ }^{*} p<0.05, * * p<0.01, * * * p<0.001$ vs controls

${ }^{\text {a }}$ Data available for 62 in the endurance group, 219 in the mixed group, 102 in the power group, 383 in the all athletes group and 201 in the control group

${ }^{\mathrm{b}}$ Data available for 220 in the mixed group, 105 in the power group and 389 in the all athletes group

SRT2D, self-reported type 2 diabetes; ST2D, screen-detected type 2 diabetes; T2D, type 2 diabetes (self-reported and screen-detected type 2 diabetes combined) 
95\% CI 0.20, 0.76). Age-adjustment did not influence the result.

The prevalence of type 2 diabetes decreased by $2.1 \%$ (OR $0.98,95 \%$ CI $0.97,0.99$ ) for every $1 \mathrm{MET}-\mathrm{h} /$ week increase in LTPA volume. This association remained significant after adjustment for age, BMI and athletic groups.

There was no significant association between the volume of LTPA and the prevalence of IFG or IGT. The prevalence of impaired glucose regulation (IFG, IGT and type 2 diabetes combined) decreased significantly when the volume of LTPA increased. Every 1 MET-h/week increase in LTPA was associated with a decrease in the prevalence of impaired glucose regulation of $0.9 \%$ (OR $0.99,95 \%$ CI $0.98,0.997)$. Adjusting separately for age or athletic group did not influence the findings. However, adjustment for BMI made these associations non-significant. The prevalence of impairment in glucose regulation according to category of LTPA volume is shown in Fig. 1.

\section{Discussion}

According to our findings the male former elite athletes with a history of vigorous physical activity had a lower prevalence of type 2 diabetes than the matched controls. Furthermore, those participants with the most LTPA in later life had the lowest prevalence of type 2 diabetes. These results support previous findings in the same cohort reporting that current LTPA level is associated with a lower risk of type 2 diabetes, and that with ageing the former athletes maintained their physically active lifestyle better than the controls [6].

With respect to type 2 diabetes the results of the present study are consistent with several previous findings regarding the beneficial effects of regular physical activity [2, 3, 7]. LTPA (both aerobic and resistance exercise) plays an important role in the prevention of type 2 diabetes [3, 7]. Various mechanisms underlie the protective effects of physical activity; for example, more advantageous body composition and higher proportion of insulin-sensitive muscle fibres.

Our results are consistent with previous reports [8, 9] that, unlike IFG, IGT increases with age. Ageing is associated with a loss of muscle mass and increased muscle weakness which, combined with a sedentary lifestyle, lead to a substantial impairment of muscle function [9].

Our study is subject to certain limitations. Typically, the validity and reliability of self-reported questionnaires are better if completed in groups rather than by an individual and give both higher and lower values than when directly measured [10]. In our study, information on smoking habits and later life LTPA were self-reported. Also, one of the study limitations is that we did not take into consideration the participants' dietary habits. Non-participants were significantly older than the participants, and they had significantly more often a reimbursable diabetes medication than the participants, suggesting that our participants are not fully representative of the population. It is also important to keep in mind that it is likely that the former elite athletes represent a genetically selected group.
Fig. 1 The percentage of participants belonging to different categories of impaired glucose regulation according to LTPA categories. (a) Normal glucose tolerance (NGT; $n=212$, $p=0.005)$, (b) type 2 diabetes $(n=124, p<0.001),(\mathbf{c}) \mathrm{IFG}$ $(n=123, p=0.378)$, (d) IGT $(n=123, p=0.004)$. MET I, $\leq 6.0$ MET-h/week; MET II, 6.1-12.0 MET-h/week; MET-III, 12.1-22.5 MET-h/week; MET IV, 22.6-45.0 MET-h/week; MET V, $\geq 45.1 \mathrm{MET}-\mathrm{h} /$ week
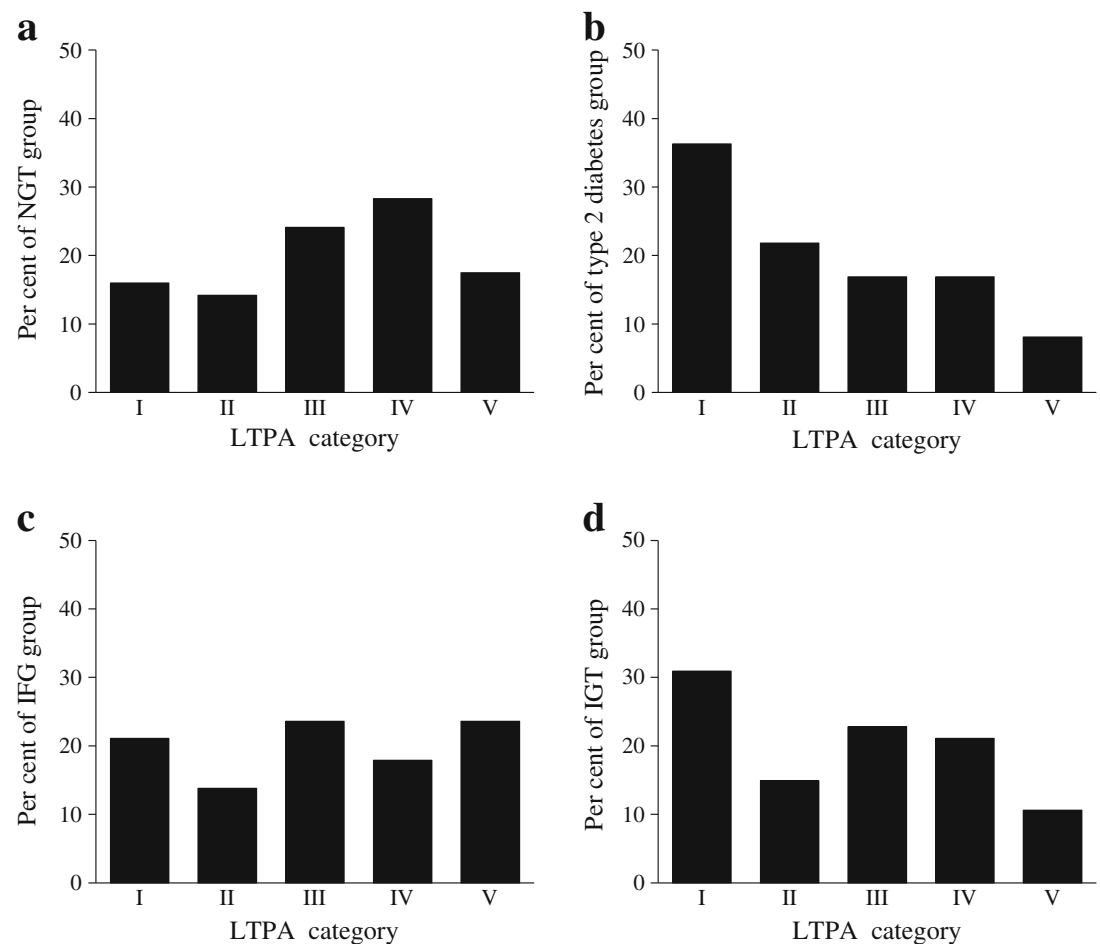
According to our study findings, being a former elite athlete is associated with a low risk of type 2 diabetes in later life. Furthermore, a high volume of current LTPA is associated with a significantly lower prevalence of type 2 diabetes. The former elite athletes also had a significantly lower prevalence of IGT than the age- and area-matched controls.

Acknowledgements We would like to thank the National Institute for Health and Welfare, Department of Public Health, University of Helsinki; Sports \& Exercise Medicine Department of Health Sciences, University of Jyväskylä; Paavo Nurmi Centre, Turku and ORTON Research Institute, Invalid Foundation, Helsinki for collaboration during the large epidemiological and clinical research programme in the year 2008. The authors thank E. Komulainen (Faculty of Behavioural Sciences, University of Helsinki, Helsinki, Finland) for help with the statistical analysis.

Funding The study was funded by the Ministry of Education and Culture, the Juho Vainio Foundation, the Finnish Heart Research Foundation, the Paavo Nurmi Foundation, the Finnish Cultural Foundation and by a grant from Medical Society of Finland, Finska Läkaresällskapet.

Duality of interest The authors have no relevant duality of interest to disclose.

Contribution statement MKL wrote the manuscript and researched data. JGE, UMK, JK, HMB and SS contributed to study design and data collection and edited the manuscript. NSW edited the manuscript and assisted with the statistical analysis. MP contributed to study design and data collection. In addition, MKL, JGE, UMK, NSW, JK, HMB, MP, THM and SS contributed to the interpretation of the results and to the discussion, reviewed the paper and approved the final version of the manuscript.

\section{References}

1. WHO (2011) Description of the global burden of NCDs, their risk factors and determinants. In: Global status report on noncommunicable diseases 2010, WHO, Geneva, pp 16-25

2. Kujala U, Kaprio J, Taimela S, Sarna S (1994) Prevalence of diabetes, hypertension, and ischemic heart diseases in former elite athletes. Metabolism 43:1255-1260

3. Lindström J, Peltonen M, Eriksson JG et al (2013) Improved lifestyle and decreased diabetes risk over 13 years: long-term follow-up of the randomised Finnish Diabetes Prevention Study (DPS). Diabetologia 5:284-293

4. The Social Insurance Institution of Finland (2013) Reimbursements for medicine expenses. Available from http:/www.kela.fi/web/en/ reimbursements-for-medicine-expences, accessed 23 October 2013

5. WHO (1999) Definition, diagnosis and classification of diabetes mellitus and its complications. Report of World Health Organization Consultation. Part 1: Diagnosis and classification of diabetes mellitus. WHO, Geneva

6. Sarna S, Kaprio J, Kujala UM, Koskenvuo M (1997) Health status of former elite athletes. The Finnish experience. Ageing Clin Exp Res 9: $35-41$

7. Bassuk SS, Manson JE (2005) Epidemiological evidence for the role of physical activity in reducing risk of type 2 diabetes and cardiovascular disease. J Appl Physiol 99:1193-1204

8. The DECODE Study Group (2005) Are insulin resistance, impaired fasting glucose and impaired glucose tolerance all equally strongly related to age? Diabet Med 22:1476-1481

9. Unwin N, Shaw J, Zimmet P, Alberti GMM (2002) Impaired glucose tolerance and impaired fasting glycaemia: the current status on definition and intervention. Diabet Med 19:708-723

10. Haskell WL (2012) Physical activity by self-report: a brief history and future issues. J Phys Act Health 9(suppl 1):S5-S10 